\title{
A Decentralized, Flat-Structured Control System for Chiller Plants ${ }^{+}$
}

\author{
Dong $\mathrm{He}^{1,2, * \mathbb{C}}$, Qingyu Xiong ${ }^{3, *}$, Xuyang Zhang ${ }^{4} \mathbb{D}$, Yunchuang Dai ${ }^{5}$ and Ziyan Jiang ${ }^{5}$ \\ College of Automation, Chongqing University, Chongqing 400044, China \\ TongFang Technovator Chongqing Co., Ltd, Chongqing 400044, China \\ College of Software, Chongqing University, Chongqing 400044, China \\ 4 Clean Energy Research Institute, College of Engineering, University of Miami, Coral Gables, FL 33146, USA; \\ x.zhang18@umiami.edu \\ 5 Building Energy Research Centre, Tsinghua University, Beijing 100084, China; \\ daiyc@mail.tsinghua.edu.cn (Y.D.); jiangzy@tsinghua.edu.cn (Z.J.) \\ * Correspondence: hedfly@163.com (D.H.); xiong03@cqu.edu.cn (Q.X.); Tel.: +86-023-6510-6238 (Q.X.) \\ + This paper is an extended version of paper published in the 2017 International Conference on Future \\ Buildings and Districts-Energy Efficiency from Nano to Urban Scale, CISBAT 2017 held in Lausanne, \\ Switzerland, 6-8 September 2017.
}

Received: 19 September 2019; Accepted: 7 November 2019; Published: 10 November 2019

Featured Application: This work can be widely used in different heating, ventilation, air-conditioning, and cooling system, helping to quickly build an efficient, stable and energy-saving control system.

\begin{abstract}
This paper presents a novel control system for chiller plants that is decentralized and flat-structured. Each device in chiller plant system is fitted with a smart node. It is a smart agent, which collects, handles and sends out information to its neighbours. All the smart nodes form a network that can realize self-organization and self-recognition. Different kinds of control strategies can be converted into series of decentralized computing processes carried on by the smart nodes. The principle and mechanism of this decentralized, flat-structured control system for chiller plants are described in detail. Then a case study is presented to show how to build the decentralized, flat-structured control system actually. The measured data shows that the decentralized control method is energy efficiency. Moreover, it is much more flexible and scalable compared with the traditional centralized control method.
\end{abstract}

Keywords: chiller plants; decentralized control; smart device

\section{Introduction}

Heating, ventilation, and air conditioning (HVAC) systems usually are the largest energy use systems in commercial buildings. For a generic office building, more than $60 \%$ of total energy use can be generated by HVAC system [1]. Within the HVAC system itself, the most energy-consuming part is the chiller plant system, which consists of chillers, pumps, cooling towers and some other electromechanical equipment. In most practical applications, considering standby capacity, operational flexibility and, most importantly, high energy efficiency under partial load, multiple devices of the same type are usually connected in parallel to a group or station. Therefore, the group operation of the pump, chiller and cooling tower systems come to be a significant topic in the optimal control of a chiller plant system, where the key points and difficulties are how to determine loading/unloading and matching speed or part load ratio (PLR) of all running equipment. 
Considerable studies have been undertaken to regulate the optimal group operation of the pump, cooling tower and chiller systems, to mainly realize the energy conservation [2] and thermal comfort goal [3]. Most researchers have focused on employing various numerical algorithms to resolve such an optimization problem. Some researchers have adopted genetic algorithm [4], dynamic programming algorithm [5], evolutionary algorithm [6], extreme value analysis algorithm [7], and mixer integer nonlinear programming (MINLP) algorithm [8] to optimize the group operation of variable speed pumps (VSPs). For the chillers' optimal group operation, evolutionary algorithm [9], generalized reduced gradient algorithm [10], branch and bound algorithm [11], particle swarm algorithm [12], and simulated annealing algorithm [13] has been adopted for the optimal chiller load (OCL). The optimization algorithms for the group operation of cooling tower include constrained inverse optimization [14], mixed-integer nonlinear programming (MINLP) algorithm [15], artificial bee colony algorithm [16], particle swarm algorithm [17].

However, these algorithms are not straightforward and usually need either the input of abundant data for the algorithm training or case-by-case development for unequal systems. Unfortunately, high computation and implementation cost make it difficult for these algorithms to be widely adopted in real applications [18].

Some researchers have tried to find analytical solutions to solve this optimization problem for assisting with the straightforward control algorithm design. An analytical solution for speed optimization has been derived [18-20]. One basic rule is to maintain the same speed ratio for identical parallel connected pumps, which has been verified by experimental studies [21,22]. Typical principles of chiller sequencing control, commonly used in practical applications, include direct power-based control, bypass flow-based control, return chilled-water temperature-based control, and total cooling load-based control [23].

In the current practical applications, whatever optimal group operation is used, the system structure has remained unchanged for almost 30 years [24]. It uses a hierarchical tree topology, as shown in Figure 1, with a centralized controller (PLC or DDC) to complete control tasks. The centralized controller firstly collect data from each unit of equipment and then perform calculations. After that, it make decisions based on a centralized control algorithm and send out control instructions to each unit of equipment. With this centralized control structure, when the system configuration changes, the control algorithm or strategy needs to be adjusted or reprogrammed accordingly. These works which include onsite measuring, wiring, configuring, commissioning, and secondary development mean that each control system is unique and case-by-case developing. Therefore, the development of a centralized chiller plant control system leads to a high labor cost.

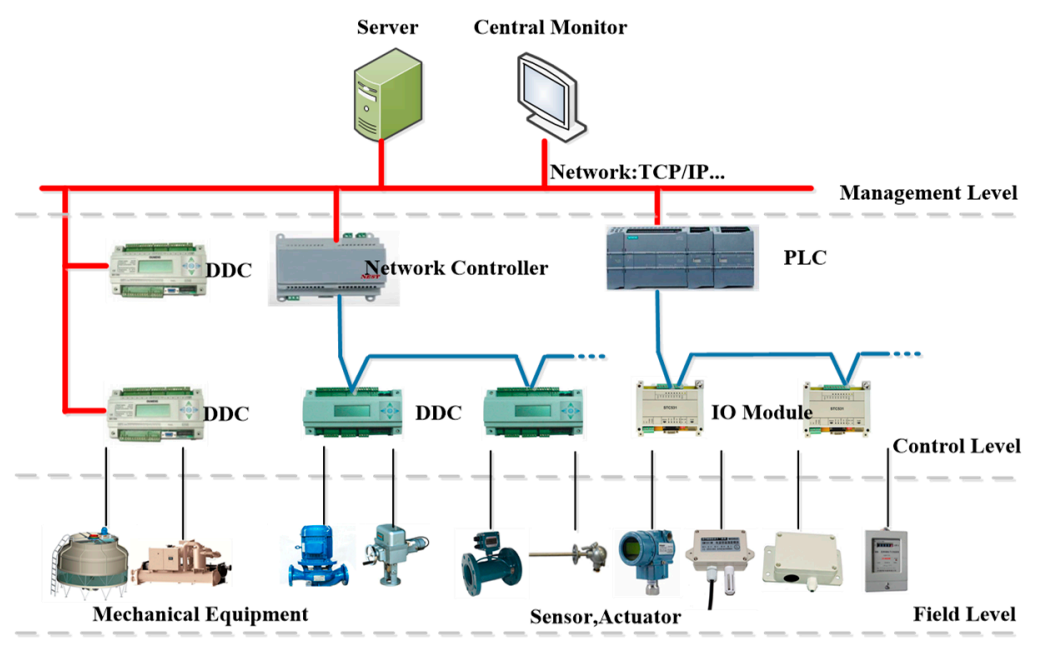

Figure 1. Typical control system structure for chiller plant system. 
To address the actual application problems described in above, this paper proposes a decentralized control method for the group control of parallel-connected device in chiller plant system. Thus, it presents a new chiller plant control system from a different point of view. Table 1 summarizes frequently used notations in this paper. The rest of this paper is organized as follows. Based on new understanding, Section 2 introduces the structure and basic elements of the decentralized, flat-structured control system. Section 3 gives a brief introduction to the decentralized optimal control algorithm for the main equipment in chiller plant system. Section 4 presents a use case to demonstrate the practicability and energy saving effect of the proposed method. Finally, Section 5 gives a conclusion, and it also presents further research directions in future.

\section{Decentralized, Flat-Structured HVAC Automation System}

Figure 2 shows that cooling tower group, VSP group, and chiller group are connected in parallel in this decentralized control structure. Each unit has its own decentralized controller, so it turns into a smart unit. These smart units for the same group are communicated by either wire or wireless, in order to meet the control requirement and increase the energy efficiency.

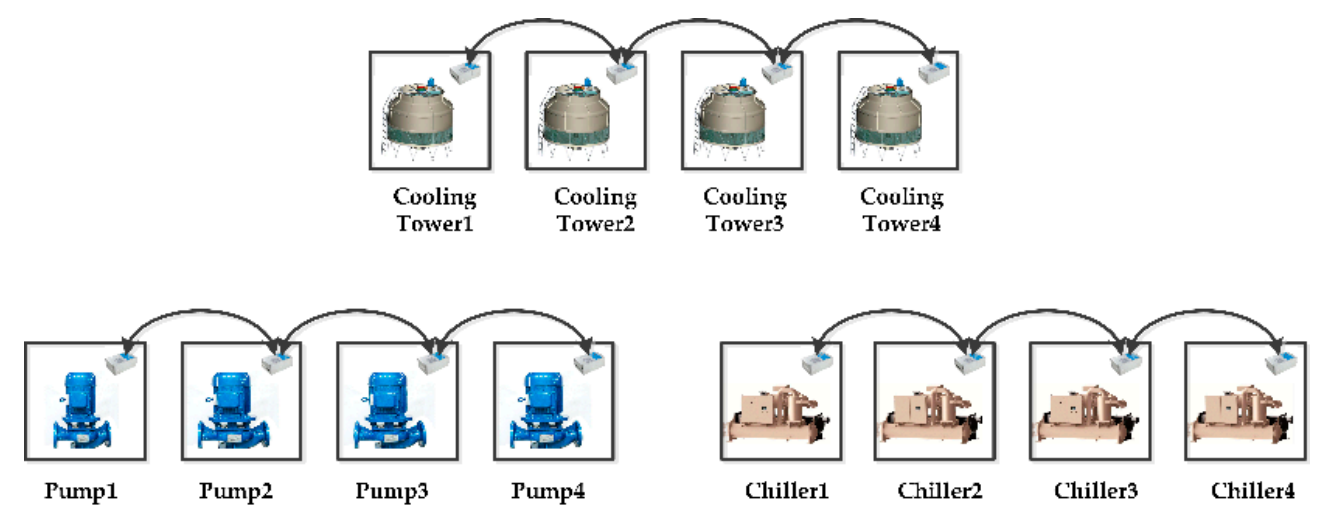

Figure 2. Structure of decentralized chiller plant control system.

Centralized controllers, such as DDC or PLC, are not used in the decentralized control system. The installation of a decentralized controller which includes the control algorithm and performance specifications can convert a conventional mechanical unit into a smart unit. As a result, this simplifies the onsite modeling, configuration, commissioning, and development, since it brings a good communication capability between different smart unites when building the whole system. These smart units will be converted into mature products integrating large amounts of preceding work by such a decentralized control structure. In addition, no extra controller is needed for the system. The decentralized control system enables units to conduct a "plug-and-play" work, and then it leads to a more flexible and scalable system than the traditional system.

\subsection{Basic Unit}

Based on the decentralized chiller plant control structure, the devices' operation mode is changed from manual configuration and centralized monitoring in the central controller to independent collaborative work between intelligent devices. Therefore, it is necessary to unify and standardize the input and output parameters of various intelligent devices. The basic work is to determine the standard information model of the smart devices.

\subsubsection{Standardized Information Model}

The establishment of the standardized information model of intelligent equipment, facing the field layer at the bottom of the decentralized control system, is the basis for universal replacement and plug-and-play implementation of intelligent devices from different manufacturers and with different 
types, as shown in Figure 3. The standardized information model of smart devices is a universal set of information for maximizing operation of similar electromechanical devices. When equipment of different manufacturers and different types changes, or when an accessory sensor or an auxiliary actuator inside the smart device changes, the smart device still follows a standardized information model, independent of the data format and communication protocol within the smart device. Thus, the decentralized control system can be directly compatible with various intelligent devices that follow the standard information model, and avoid a large number of networking configuration work on site.

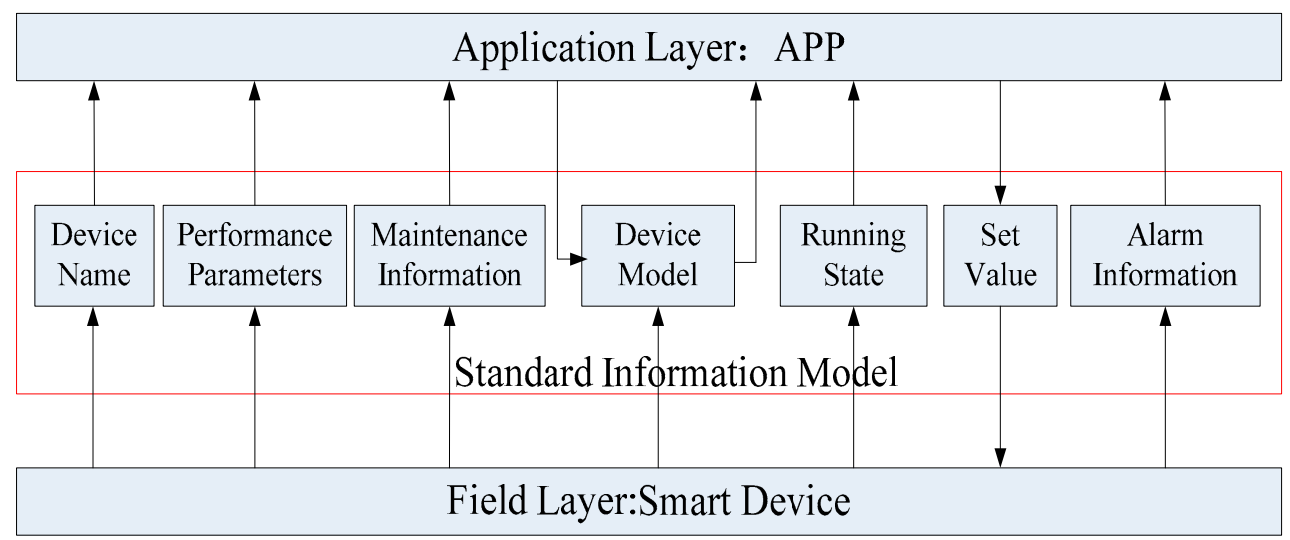

Figure 3. The application of standard information model.

The establishment of the standardized information model of intelligent devices, facing the application layer at the upper layer of the decentralized control system, provides the basis for the "universal" development and application of various management software and control algorithms, as shown in Figure 3. Based on the standardized information model of various types of intelligent devices, the data interfaces provided by various standard information models can be called, so that the APPs at the application layer can be directly operated on the decentralized control system of different chiller plant system without secondary development for different systems. It is the basis for the decentralized control algorithm and the versatility and extensibility of other APPs in the application layer.

In summary, the standardized information model of smart devices is the basis for the versatility and extensibility of the decentralized control system. The information of the smart device in its actual operation and the cooperation between the smart devices can be divided into seven categories: device name, maintenance information, performance parameters, device model, running status, set value, and alarm information, as shown in Figure 3. At the same time, according to the seven information classification methods, taking the chiller, water pump and cooling tower commonly used in the chiller plant system as an example, all the information points of each smart device are classified, and all the information points in each category are listed in Table 1.

The data sources of these seven types of parameters are different. Some are built into the smart device by the device manufacturer at the factory, such as device model, maintenance information, etc. Some are obtained by the device's own controller DCU (Drive Control Unit) in the actual operation, and then they are mapped and updated to the standard information model database in decentralized controller, such as various operating status information during the running of the equipment, alarm information, etc. Some are the set value that are obtained through coordinated calculation by the management software or the control algorithms running at the decentralized controller. And some are the device name or the device number derived from the identification rule running at the decentralized controller level. 
Table 1. Standard information points of the smart device.

\begin{tabular}{|c|c|c|c|c|}
\hline Name & $\begin{array}{l}\text { Performance } \\
\text { Parameter }\end{array}$ & Device Model & Operating Status & Set Value \\
\hline Chiller & $\begin{array}{l}\text { Rated cooling capacity, } \\
\text { rated power, rated flow } \\
\text { rate of freezing/cooling } \\
\text { water, lower limit of } \\
\text { freezing/cooling water } \\
\text { flow, duration of on/off } \\
\text { protection }\end{array}$ & $\begin{array}{l}\text { a1, a2, a3, a4, a5, a6, } \\
\text { a7, a8, a9, a10 }\end{array}$ & $\begin{array}{l}\text { Automatic/manual state, start } \\
\text { and stop state, cumulative } \\
\text { running time, number of starts, } \\
\text { cold COP, cold current ratio, } \\
\text { evaporator inlet and outlet } \\
\text { water temperature, condenser } \\
\text { inlet and outlet water } \\
\text { temperature, evaporation } \\
\text { temperature, condensation } \\
\text { temperature, evaporation } \\
\text { pressure, condensing pressure, } \\
\text { compressor Import and export } \\
\text { temperature, water flow } \\
\text { switch signal, water side valve } \\
\text { status }\end{array}$ & $\begin{array}{l}\text { Automatic/manual state, } \\
\text { start/stop state, chilled water } \\
\text { outlet water temperature }\end{array}$ \\
\hline Cooling tower & $\begin{array}{l}\text { Rated water quantity, } \\
\text { rated air volume, rated } \\
\text { power, on/off } \\
\text { protection duration }\end{array}$ & $\mathrm{a} 1, \mathrm{a} 2, \mathrm{a} 3, \mathrm{a} 4$ & $\begin{array}{l}\text { Automatic/manual status, fan } \\
\text { start and stop status, } \\
\text { accumulated running time, } \\
\text { number of starts, fan speed, } \\
\text { fan power, ambient wet bulb } \\
\text { temperature, water valve inlet } \\
\text { and outlet status, inlet and } \\
\text { outlet water temperature }\end{array}$ & $\begin{array}{l}\text { Automatic/manual state, } \\
\text { start/stop state, frequency, } \\
\text { outlet water temperature, } \\
\text { upper and lower limits of fan } \\
\text { operation, lower limit of } \\
\text { cooling water flow }\end{array}$ \\
\hline
\end{tabular}

\subsubsection{Device Model}

Chiller. COP is usually used to express the operating efficiency of the chiller, i.e., the ratio of cooling capacity to power consumption of the chiller. Generally, it can be fitted as a quadratic polynomial of three variables [25], i.e., a ten-coefficient formula commonly used by chiller manufacturers. The specific expression is shown in Equation (1),

$$
\mathrm{COP}=a_{1}+a_{2} \cdot P L R+a_{3} \cdot T_{\text {lea }}+a_{4} \cdot T_{\text {ent }}+a_{5} \cdot P L R^{2}+a_{6} \cdot T_{\text {lea }}^{2}+a_{7} \cdot T_{\text {ent }}^{2}+a_{8} \cdot P L R \cdot T_{\text {lea }}+a_{9} \cdot P L R \cdot T_{\text {ent }}+a_{10} \cdot T_{\text {lea }} \cdot T_{\text {ent }}
$$

where PLR is the partial load ratio, $T_{\text {lea }}$ is the temperature of the water leaving the evaporator, and $T_{\text {ent }}$ is the temperature of the water entering the condenser.

Pump. Firstly, a new variable, pump speed ratio $w(0<w<1)$, is defined as

$$
w=n_{\text {act }} / n_{\text {rat }}
$$

where $n_{\text {act }}$ is the actual running speed of pump, $n_{\text {rat }}$ is the rated speed of pump.

Then, the pumps operation curve at all rated and non-rated working conditions could be expressed as Equations (3) and (4),

$$
\begin{gathered}
H=b Q^{2}+c w Q+d w^{2} \\
\eta=j(Q / w)^{2}+k(Q / w)+l
\end{gathered}
$$

where $H, Q$, and $\eta$ are the pump head, flow rate and mechanical efficiency at the rated speed, respectively; $b, c, d$ and $j, k, l$ are constants that vary with the characteristics of a specific pump, which can be obtained from the experimental data or manufacturer data under the rated conditions.

Cooling tower. Under normal circumstances, the cooling tower efficiency and the ratio between the condenser water mass flow rate and the air mass flow rate of the cooling tower can be combined into a polynomial curve [26], as shown in Equation (5),

$$
\eta=s_{1}+s_{2} \cdot \frac{m_{\text {wat }}}{m_{\text {air }}}+s_{3} \cdot\left(\frac{m_{\text {wat }}}{m_{\text {air }}}\right)^{2}+s_{4} \cdot\left(\frac{m_{\text {wat }}}{m_{\text {air }}}\right)^{3}
$$


where $\eta, m_{\text {wat }}, m_{\text {air }}$ are the cooling tower efficiency, condenser water mass flow rate and air mass flow rate respectively; $s_{1}, s_{2}, s_{3}, s_{4}$ are constants that vary with the characteristics of a specific cooling tower, which can be obtained from the manufacturer data or experimental data under the rated conditions.

The device manufacturers can fit the corresponding coefficients according to corresponding equation, based on the test data of multiple working conditions, by testing the cold machine under different working conditions, and write the coefficients into the device before the device leaves the factory.

\subsection{Network \& Communication}

Under the consideration of the designing and using functions, the electromechanical devices in the chiller plant system are physically connected through pipes according to a certain paradigm, which can also reflect the control requirements between the devices to some extent. As shown in Figure 4a, it is a schematic diagram of the physical connection of a typical cooling station. Four refrigerating pumps are connected in parallel, that is, the adjacent refrigerating pumps are directly connected by water pipes. In operation, four pumps need to mutually negotiate to complete the control objectives. Therefore, the basic simple and intuitive rule is to connect the devices with communication lines according to the physical connection topology of the devices. The communication connection topology of the smart device shown in Figure $4 \mathrm{~b}$ is identical to the physical connection topology of the device.

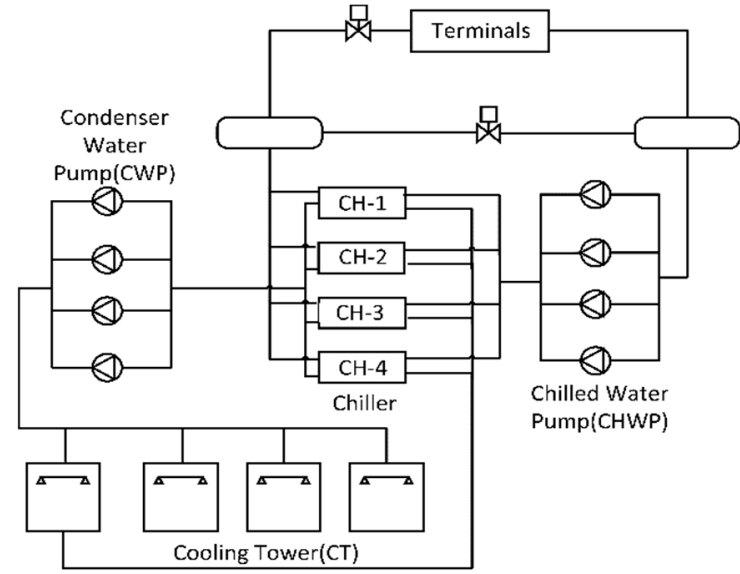

(a)

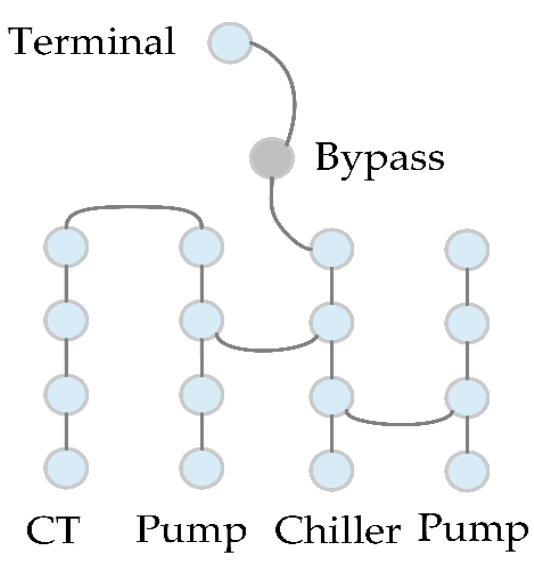

(b)

Figure 4. (a) Physical connection topology of the device; (b) Communication connection topology of the device.

The special features of the above network are: (1) there is no centre of the network, all nodes are equal; (2) nodes can only communicate with their neighbours; (3) the network is scalable. It's very flexible to expand the network by connecting nodes to the adjacent nodes in the network.

Based on the simple and straightforward rules, the communication connection is completed. The "smart devices" exchange information through communication to complete various control tasks together, which is equivalent to automatically generating the control system, that is, completing the "plug and play" system construction.

\subsection{Decentralized Computing}

Since there is no centre node in the system, decentralized computing is required. In a traditional system, in which the centre node collects data, computing is carried out by the centre node, like the brain. However, in the decentralized system, computing is carried out through collaboration of all the nodes, like the neuron system. For example, in solving a linear system of equation $A x=b$, the problem decomposition and decentralized algorithm are as follows [27]: 
First, the problem is decomposed into pieces. Each node holds part of the problem. Back to the chiller plant system, the parameters describe whether the pieces of the problem come from local measurement or a neighbour of the basic unit. No node knows the entire problem. Each node corresponds to an unknown in the system of equations, as well as a row equation. Second, based on the variable information and local variables of the adjacent nodes and the equation information, each node updates the calculated local variables $x_{i}=b_{i}-\sum a_{i j} x_{j}$. Through repeated interactions between a large number of nodes, the results of each node's calculations converge. In this process, a large number of identical individuals work together to accomplish a global problem by performing local tasks and collaborating.

\section{Decentralized Control Algorithm}

\subsection{System Cooperative Protection Algorithm}

This algorithm focuses on the safe and stable operation of all intelligent electromechanical equipment (chiller, water pump, cooling tower) in the cooling station, ensuring that it can work independently. The basic purpose is to automatically realize the automatic sequence start and sequence stop of each device of the cooling station system. During the whole running process, after the user sets the switch of the device through the schedule or manually, the device interacts with other devices in the same group to determine whether the condition for executing the instruction is met. If the condition is met, the corresponding power on/off command is executed; if the conditions are not met, the status is maintained and the instruction decision judgment is continued.

\subsection{Device Self-Organizing Optimization Control Algorithm}

The adjustment process of the device self-organizing optimization control algorithm is an iterative process in which multiple devices negotiate with each other. The basic principle of optimal control is that all equipment takes the highest point of efficiency as the starting point of adjustment, and determines the direction of adjustment according to the direction in which the initial working point of the equipment deviates from the highest efficiency point, and at the same time, transmits the expected efficiency $\varepsilon$ and demand deviation $\Delta$ to the neighbor nodes. According to the received efficiency expectation and demand deviation, the node combines its current efficiency expectation, outputs new efficiency expectation $\varepsilon_{\text {out }}$ and demand deviation $\Delta_{\text {out }}$, and transmits it to the neighbor nodes. When the transmitted demand deviation is less than the set calculation precision $\delta$, the calculation converges. Then the nodes output and execute the new control results. The flow chart of the device self-organizing optimization control is shown in Figure 5.

For different devices, $\varepsilon$ and $\Delta$ need to be defined separately. The chiller efficiency expectation $\varepsilon$ can be defined as the ratio of the current COP to the highest COP, and the deviation $\Delta$ of the distribution amount is the deviation between the current assumed cooling load and the total system cooling load demand.

The pump efficiency expectation $\varepsilon$ can be defined as the ratio of the current efficiency to the highest efficiency, and the deviation $\Delta$ of the distribution amount is the deviation of the currently provided flow from the total system flow demand.

In actual engineering, in order to avoid uneven water distribution, the lower limit of the flow rate of the cooling tower is usually set to avoid uneven flow of the flow due to the flow rate of the cooling tower. Therefore, the number optimization problem of the cooling tower can be simplified as follows, the cooling towers are turned on as much as possible under the given total flow rate of the condenser water, and the flow rate assigned to each cooling tower is not lower than the minimum flow rate set by each. Therefore, the efficiency expectation $\varepsilon$ can be defined as the ratio of the actual condenser water flow rate $G$ of the cooling tower to the minimum condenser water flow rate $G_{\min }$ set by the cooling tower, i.e., $\varepsilon=G / G_{\min }$, and the deviation $\Delta$ of the distribution amount is the difference between the total flow taken by the current cooling tower and the total flow of the system condenser water. More 
detailed description of the device self-organizing optimization control algorithm can be referred to article [28].

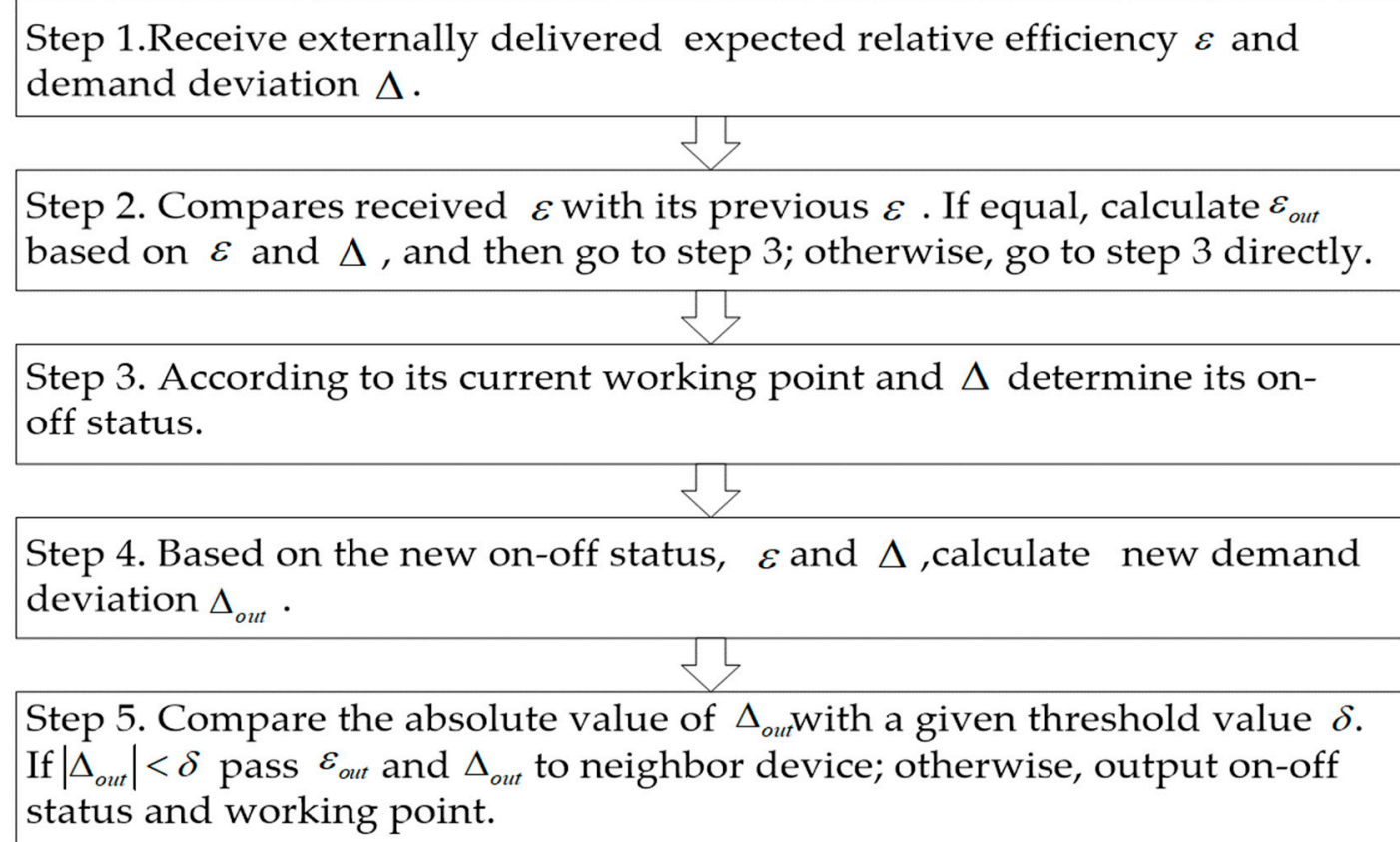

Figure 5. The flow chart of the device self-organizing optimization control.

\subsection{Coordination Optimization Algorithm for Trunk Pipe Coordinator}

The function of the chilled water trunk pipe coordination controller optimization control is to calculate the user side cooling load demand, and at the same time, by adjusting the bypass valve, the chilled water quantity is guaranteed to meet the minimum flow demand of the chiller. According to the minimum flow demand of the chiller, the current chilled water flow, and the measured value of the pump differential pressure, the demand differential pressure that meets the minimum flow rate is calculated. When the demand pressure difference is greater than the set value of the differential pressure of the water pump, the opening degree of the bypass valve is increased; when the pressure difference is less than the set value of the differential pressure of the water pump, the opening degree of the bypass valve is reduced. Through the combination with the control of the chilled water pump, the amount of chilled water is satisfied while minimizing the energy loss caused by the bypass.

The function of the condenser water trunk pipe coordination controller is to ensure that the return water temperature of the chiller is not less than the lower limit of the return water temperature. When it is found that the return water temperature of the condenser water is lower than the lowest temperature of the chiller operation, increase the opening degree of the bypass valve to ensure the normal operation of the unit; when the return water temperature is higher than the lowest temperature of the chiller operation, gradually reduce the opening degree of the bypass valve to reduce the energy loss caused by the bypass.

\section{Case Study Based on New System}

This chapter aims to conduct a case study applying the decentralized, flat-structured HVAC automation system to evaluate the cooling system for an aquatics centre, which is a commercial building located in Beijing, China. The climate of Beijing is a typical semi-humid continental monsoon climate in the north temperate zone. It is hot and rainy in summer, cold and dry in winter, and short in spring and autumn. The annual average temperature in the main urban area is between 11 and $13^{\circ} \mathrm{C}$. The annual extreme maximum temperature usually occurs in July, generally between 35 and $40^{\circ} \mathrm{C}$, 
and the annual extreme minimum temperature usually occurs in January, generally between 20 and $-14^{\circ} \mathrm{C}$. Summer precipitation accounts for about three quarters of annual precipitation. In this case study, we demonstrate the energy efficiency, operability and cost saving of the control system.

\subsection{System Description}

The aquatics centre adopts the chiller plant system for centralized cooling, with a cooling area of about 80,000 square meters. The chiller plant system mainly includes three centrifugal chillers, one centrifugal chiller with heat recovery, six cooling towers, five condenser water pumps, five chilled water pumps. These devices are mainly installed on the underground second floor. The nominal information of the main components in the cooling system is listed in Table 2. The schematic drawing of the cooling system is shown in Figure 6.

Table 2. Nominal information of the main components in the cooling system.

\begin{tabular}{|c|c|c|c|c|}
\hline Equipment & Quantity & Nominal Equipment Information & Unit & Value \\
\hline \multirow{3}{*}{ Centrifugal Chiller } & \multirow{3}{*}{3} & Cooling Capacity & $\mathrm{kW}$ & 2813 \\
\hline & & Power & $\mathrm{kW}$ & 535 \\
\hline & & Design COP & - & 5.26 \\
\hline \multirow{3}{*}{$\begin{array}{l}\text { Heat Recovery } \\
\text { Centrifugal Chiller }\end{array}$} & \multirow{3}{*}{1} & Cooling Capacity & $\mathrm{kW}$ & 1406 \\
\hline & & Power & $\mathrm{kW}$ & 286 \\
\hline & & Design COP & - & 4.92 \\
\hline \multirow{2}{*}{ Cooling Tower } & \multirow[b]{2}{*}{6} & Power & $\mathrm{kW}$ & 22 \\
\hline & & Flowrate & $\mathrm{m}^{3} / \mathrm{h}$ & 700 \\
\hline \multirow{6}{*}{$\begin{array}{l}\text { Condenser Water } \\
\text { Pump (CWP) }\end{array}$} & \multirow{3}{*}{3} & Power & $\mathrm{kW}$ & 55 \\
\hline & & Flowrate & $\mathrm{m}^{3} / \mathrm{h}$ & 365 \\
\hline & & Head & $\mathrm{m}$ & 36.5 \\
\hline & \multirow{3}{*}{2} & Power & $\mathrm{kW}$ & 37 \\
\hline & & Flowrate & $\mathrm{m}^{3} / \mathrm{h}$ & 181 \\
\hline & & Head & $\mathrm{m}$ & 35.4 \\
\hline \multirow{6}{*}{$\begin{array}{l}\text { Chilled Water } \\
\text { Pump (CHWP) }\end{array}$} & \multirow{3}{*}{3} & Power & $\mathrm{kW}$ & 110 \\
\hline & & Flowrate & $\mathrm{m}^{3} / \mathrm{h}$ & 637 \\
\hline & & Head & $\mathrm{m}$ & 38.2 \\
\hline & \multirow{3}{*}{2} & Power & $\mathrm{kW}$ & 55 \\
\hline & & Flowrate & $\mathrm{m}^{3} / \mathrm{h}$ & 312 \\
\hline & & Head & $\mathrm{m}$ & 43.3 \\
\hline
\end{tabular}

The original automatic control system of the cooling station cannot play the role of automatic control and energy-saving operation. There are several outstanding problems: firstly, the automatic control system has been modified many times, the original system is difficult to be closed and upgraded, and the retrofitting cost is high; secondly, the automatic control system is basically abandoned in maintenance, and cannot truly realize automatic control. The number of running equipment and frequency conversion operation require manual operation, which does not reduce the labor cost and optimize the operation. 


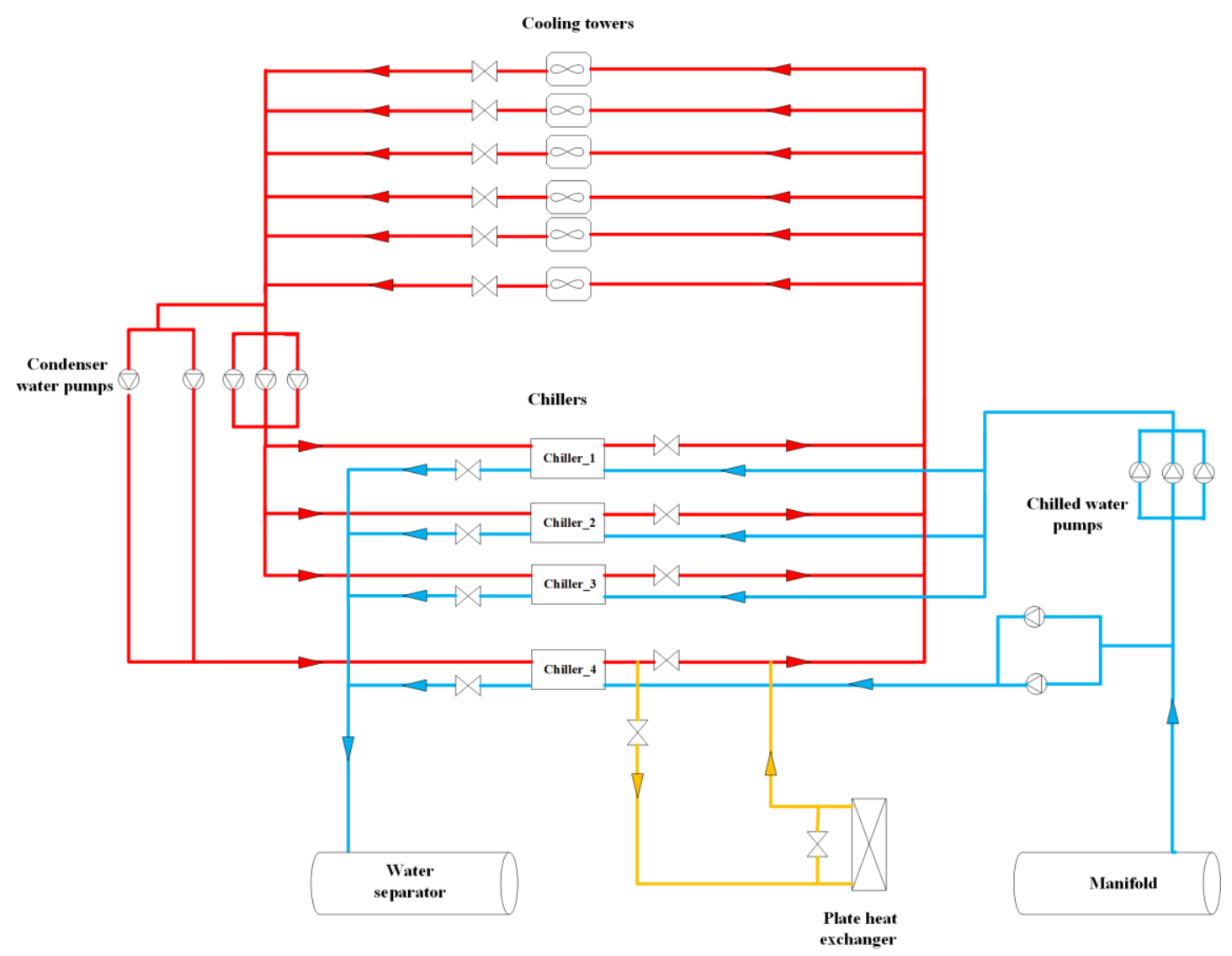

Figure 6. Schematic drawing of the cooling system.

\subsection{Scheme Design of the Decentralized Control System}

On the basis of the original water chiller, cooling tower, condenser water pump, chilled water pump and related coordination devices, an intelligent node (CPN, Computing Process Node) is set for each large electromechanical equipment, which is upgraded to intelligent electromechanical equipment. $\mathrm{CPN}$ is responsible for monitoring various parameters related to the target equipment and establishing an intelligent node network according to the physical topology relationship. The system topology connection relationship is shown in Figure 7.

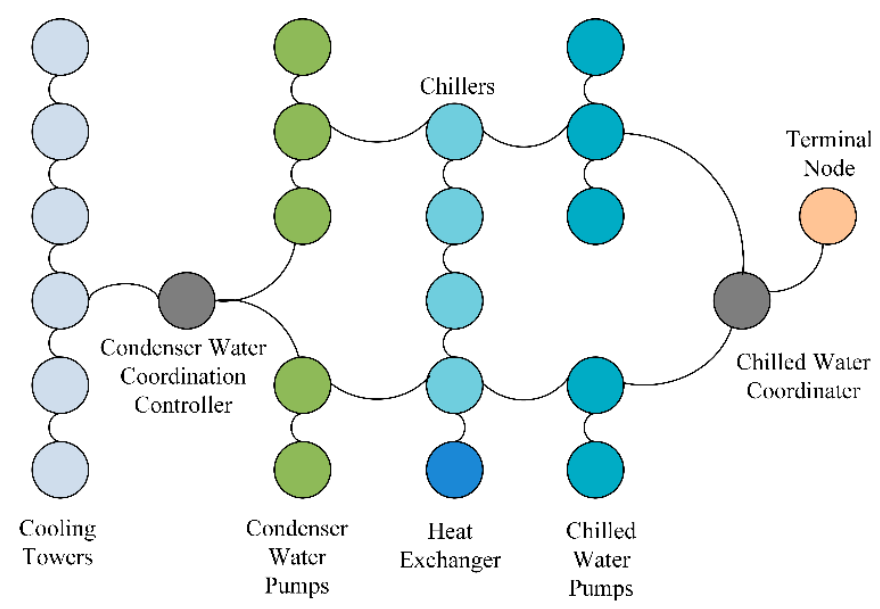

Figure 7. Topological diagram of intelligent device with decentralized control. 
All standard databases are built in each CPN. When the CPN is connected to the live DCU, through the information exchange, the CPN will know that the DCU corresponds to a chiller or a water pump, thus automatically forming a database corresponding to the DCU. The detailed information of the standard database for each device are described in Section 2.1. The model parameters of chillers and pumps are obtained from device manufacturer, which can be seen in Tables 3 and 4 . Each CPN has powerful arithmetic processing functions. All CPNs are connected to form a multicore, centerless computing platform, namely a "centralized computer."

Table 3. Chiller model parameters.

\begin{tabular}{ccccccccccc}
\hline Name & a1 & a 2 & a 3 & a 4 & a 5 & a6 & a7 & a8 & a 9 & a 10 \\
\hline Chiller1-3 & 14,317 & 9.593 & 0.413 & -0.531 & -12.038 & 0.013 & 0.003 & -0.088 & 0.217 & -0.009 \\
\hline Chiller4 & 8.918 & 15,723 & 0.534 & -0.575 & -11.767 & 0.006 & 0.006 & 0.048 & 0.074 & -0.014 \\
\hline
\end{tabular}

Table 4. Pump model parameters.

\begin{tabular}{ccccccc}
\hline Name & $\mathbf{a}$ & $\mathbf{b}$ & $\mathbf{c}$ & $\mathbf{j}$ & $\mathbf{k}$ & $\mathbf{1}$ \\
\hline CHWP01 3 & -0.0000804 & 0.02293 & 29.34 & -0.00000336 & 0.002592 & 0.2015 \\
CHWP04 5 & -0.0000478 & -0.006593 & 33.46 & -0.00000597 & 0.004493 & 0.0596 \\
CWP01 3 & -0.00008308 & 0.05683 & 24.28 & -0.00000299 & 0.003333 & -0.2459 \\
CWP04 5 & -0.001292 & 0.588085 & -36.6742 & -0.00000497 & 0.004493 & 0.0596 \\
\hline
\end{tabular}

Figure $8 \mathrm{~b}$ is the physical map of the CPNs. Each CPN is connected according to the physical topology relationship and is assembled into cabinets which are shown in Figure 8. Finally, each CPN is downloaded the corresponding control algorithm APP described in Section 3. Thus, all the CPNs can automatically monitor the parameters and issue control instructions.

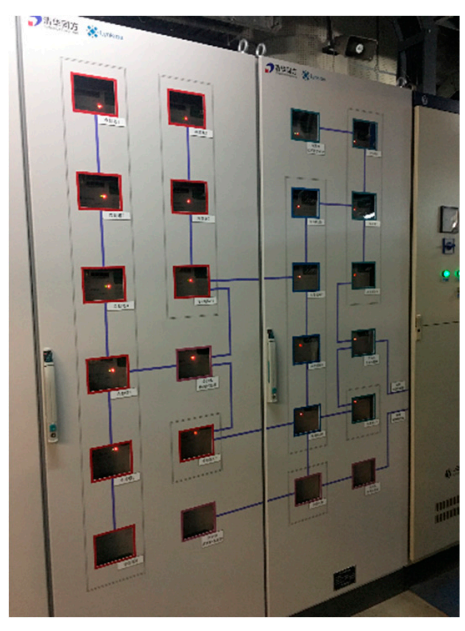

(a)

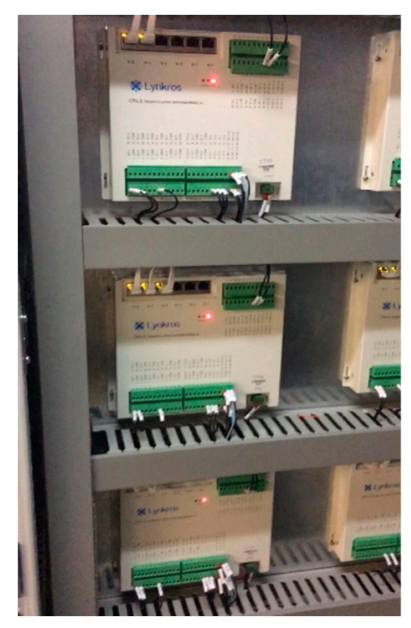

(b)

Figure 8. (a) Decentralized control cabinet; (b) Intelligent node (CPN).

\subsection{Results and Analysis}

From 19 September 2018, the system started to operate the energy-saving optimization strategy. The comparison of the operation effects of the optimization control is as follows, among which, from September 16 to 18 , the larger chiller was turned on, the water pump and cooling tower was operating at a constant frequency of $50 \mathrm{HZ}$, and the number of units was manually adjusted by the user. From September 19 to 21, the chiller was automatically selected according to the terminal load. The 
frequency and number of water pumps and cooling towers were automatically adjusted by optimizing control APP.

To analyze the energy efficiency of the decentralized control system, the energy use of the main equipment before and after the installation of the control system is calculated, which can be seen in Figure 9. Only 6-day energy use records are selected, mainly because the start time of optimal control operation is September 19, and there is little difference in meteorological conditions for these 6 consecutive days. Specific meteorological parameters are shown in the Table 5. The highest and lowest temperatures in these six days are $27^{\circ} \mathrm{C}$ and $14{ }^{\circ} \mathrm{C}$, respectively. And the weather is mainly cloudy and sunny.

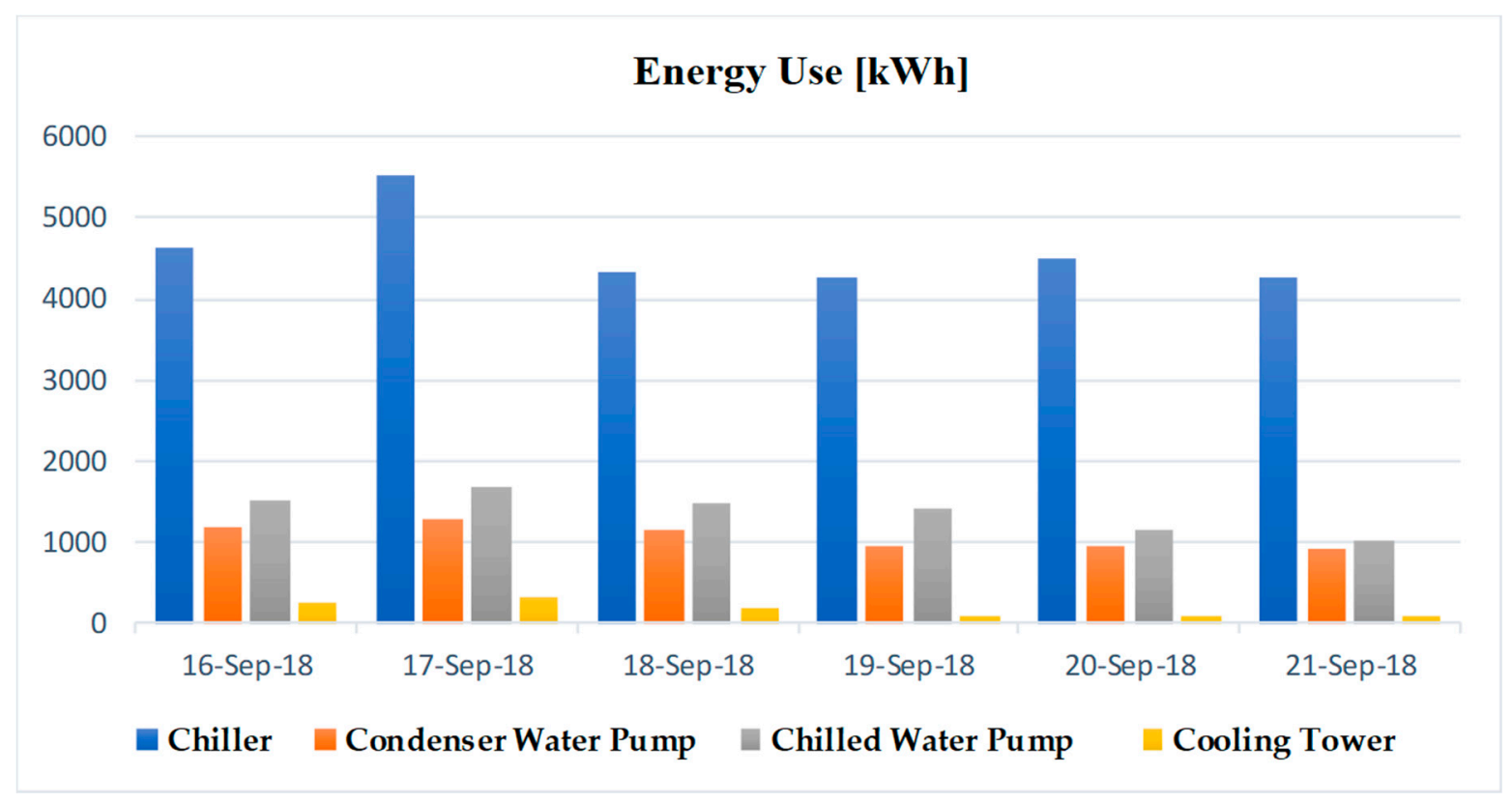

Figure 9. Energy use of the main device in statistical time period.

Table 5. Specific meteorological parameters of analysis time period.

\begin{tabular}{ccccccc}
\hline Category & $\begin{array}{c}\mathbf{1 6} \\
\text { September. }\end{array}$ & $\begin{array}{c}\mathbf{1 7} \\
\text { September }\end{array}$ & $\begin{array}{c}\mathbf{1 8} \\
\text { September }\end{array}$ & $\begin{array}{c}\mathbf{1 9} \\
\text { September }\end{array}$ & $\begin{array}{c}\mathbf{2 0} \\
\text { September }\end{array}$ & $\begin{array}{c}\mathbf{2 1} \\
\text { September }\end{array}$ \\
\hline$T_{\max }\left({ }^{\circ} \mathrm{C}\right)$ & 25 & 27 & 25 & 25 & 27 & 25 \\
$T_{\min }\left({ }^{\circ} \mathrm{C}\right)$ & 14 & 16 & 17 & 17 & 16 & 14 \\
Weather & Fine & Cloudy & Cloudy & Cloudy & Cloudy & Fine \\
\hline
\end{tabular}

Condenser water pumps and chilled water pumps were adjusted by number and frequency conversion, with energy saving rates of $20.9 \%$ and $19.6 \%$ respectively. At the same time, the original system had all cooling tower water valves fully opened. Under the automatic control strategy, the cooling tower water valves were switched on and off according to the cooling water volume and the operation of the cooling tower fan, avoiding the water mixing phenomenon of the cooling tower, which reduced energy use by $63.4 \%$. The average temperature of condenser water decreased more than $1{ }^{\circ} \mathrm{C}$, and the energy efficiency of the chiller was relatively improved $10 \%$. The comparison of the energy use of the main device under traditional control and decentralized control can be seen in Figure 10. Totally, the energy saving rates of the whole system reached to $15.4 \%$, which can be seen in Table 6 . 


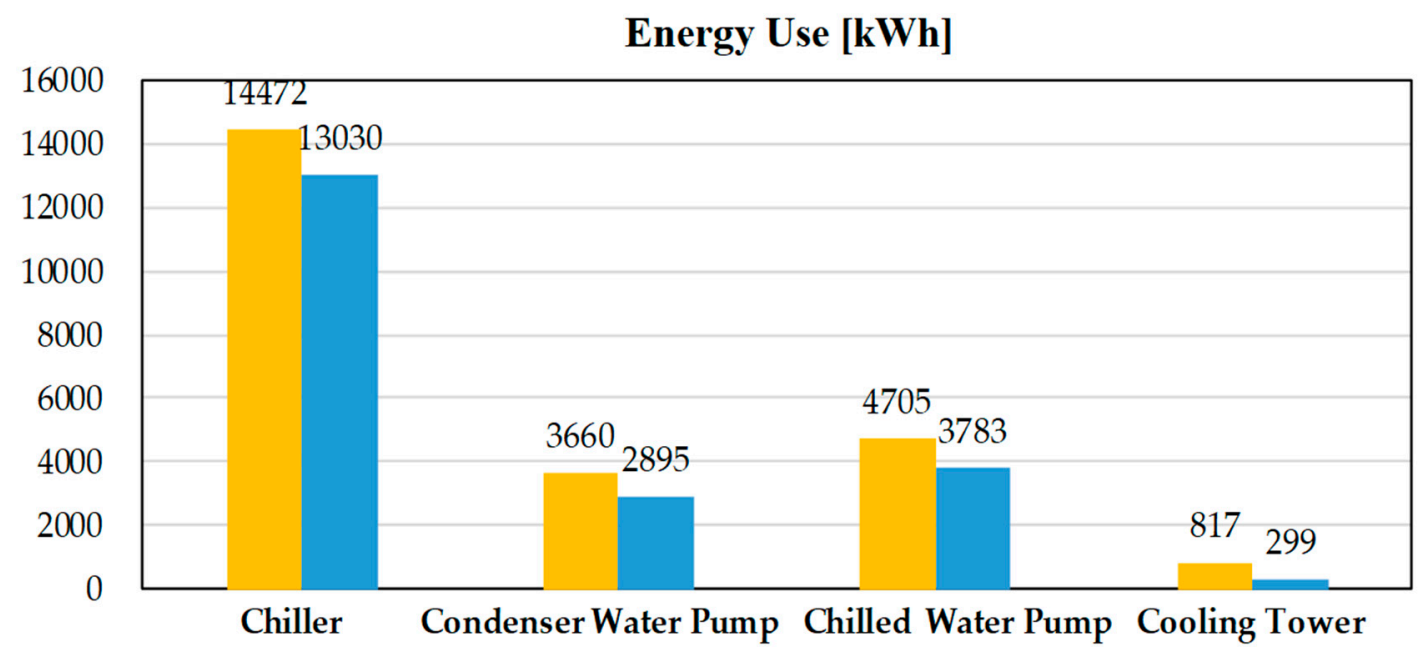

Traditional control (9.16 9.18) — Decentralized control (9.19 9.21)

Figure 10. Comparison of the energy use of the main device.

Table 6. Comparison of energy use under different control systems.

\begin{tabular}{cccc}
\hline Energy Use Category & $\begin{array}{c}\text { Traditional Control } \\
\mathbf{( 9 . 1 6} \text { 9.18) }\end{array}$ & $\begin{array}{c}\text { Decentralized Control } \\
\mathbf{( 9 . 1 9 \sim 9 . 2 1 )}\end{array}$ & Energy Saving Rate \\
\hline Chiller $(\mathrm{kWh})$ & 14,472 & 13,030 & $10 \%$ \\
Condenser Water Pump & 3660 & 2895 & $20.9 \%$ \\
(kWh) & 4705 & 3783 & $19.6 \%$ \\
Chilled Water Pump $(\mathrm{kWh})$ & 817 & 299 & $63.4 \%$ \\
Cooling Tower $(\mathrm{kWh})$ & 23,654 & 20,007 & $15.4 \%$ \\
Total $(\mathrm{kWh})$ & & & \\
\hline
\end{tabular}

In order to further reflect the energy conservation of the method proposed in this paper, I calculated the monthly system energy use of the chiller plant system from starting up in May to shutting down at the end of September. The comparison of the energy use of the main device in each month is shown in Figure 11. Table 7 lists the monthly energy use of the main device from May to September. From 19 September 2018, the system started to operate the energy-saving optimization strategy. In September, the total energy use of the chiller plant system decreased significantly compared with the previous four months, 267,953 kWh less than that in August and 33,996 kWh less than that in May.

At present, the decentralized control system has the following functions:

(1) System remote monitoring. Users with permission can browse the operation status, failure status, key operation parameters, operation energy use, etc. of each controlled device by logging in the webpage; and can control the start-stop state of each equipment according to the authority, or set the parameters such as rotation speed, frequency, chiller outlet water temperature setting value, etc.

(2) The operation and maintenance of the equipment are simplified without complicated manual operation procedures. The daily time switch machine can be set by setting the schedule. The management personnel can set and switch modes according to the scene, thus adapting to flexible and changeable management requirements.

(3) Realize the local safety protection and sequential start-stop of the equipment independently.

(4) Through coordinated control among equipment to reduce energy use in system operation. During system operation period, through energy-saving optimization APP to realize efficient and optimized operation of the system. 


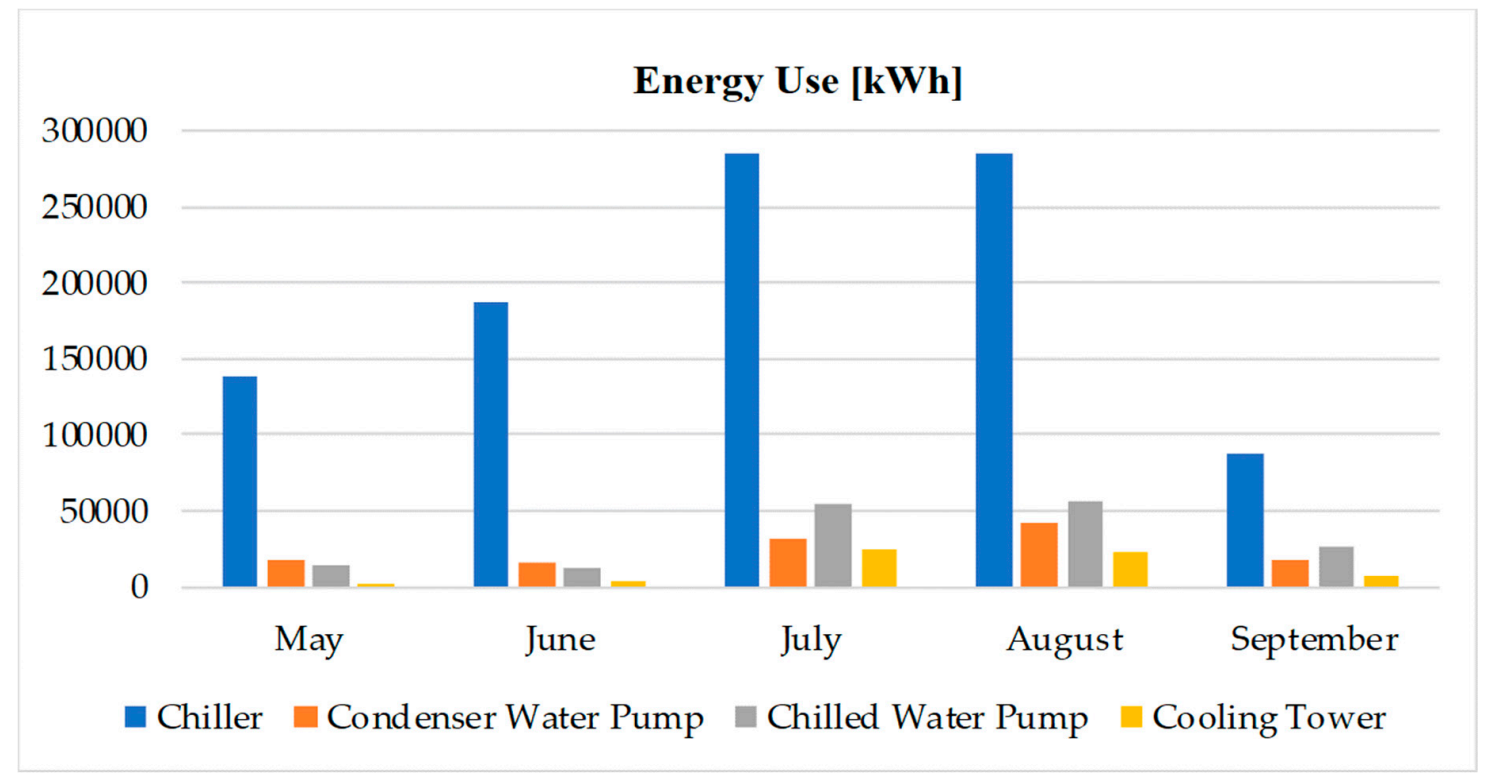

Figure 11. Comparison of the monthly energy use of the main device.

Table 7. Monthly energy use of the main device.

\begin{tabular}{cccccc}
\hline Energy Use Category & May & June & July & August & September \\
\hline Chiller $(\mathrm{kWh})$ & 139,285 & 187,243 & 284,623 & 285,242 & 87,219 \\
Condenser Water Pump $(\mathrm{kWh})$ & 16,990 & 16,004 & 32,174 & 41796 & 18,404 \\
Chilled Water Pump $(\mathrm{kWh})$ & 14,098 & 12,721 & 53776 & 56747 & 26,883 \\
Cooling Tower $(\mathrm{kWh})$ & 2751 & 3583 & 24,566 & 23296 & 6623 \\
Total $(\mathrm{kWh})$ & 173,124 & 219,551 & 395,140 & 407,081 & 139,128 \\
\hline
\end{tabular}

In the commissioning phase, the decentralized control system eliminates manual network configuration commissioning work. In the maintenance phase, the operation and maintenance of the equipment is simplified, and no complicated manual operation process is required. Thus, it is time saving and cost saving.

In summary, the proposed decentralized, flat-structured HVAC automation system is energy efficiency, operability and cost saving.

\subsection{Prospects for Optimal Control}

Optimizing the control of the terminal air-conditioning box system can facilitate the supply of cold energy on demand, reduce the bypass of water and solve the problem of "large flow and small temperature difference". At the same time, the control of the cooling station can be combined with the air conditioning box, and the set value of the total chilled water supply pressure which can be adjusted according to the terminal intelligent node. For example, if the water quantity of the terminal equipment is insufficient, the rotating speed of the water pump can be increased according to the number of terminals that do not meet the requirements. Under the condition that all ends meet the requirements, if there is too much water at the end, the rotating speed of the water pump is reduced. In this way, on the premise of meeting the demand of cooling capacity at each end, the pressure set point is reduced as much as possible to further lower the energy use of the water pump.

\section{Conclusions}

The automation system is complicated and prolonged, because it requires an enormous amount of secondary onsite development effort like configuration and commission. Each control system should be exclusive and individual development for onsite measurement, wiring, configuration, commissioning, 
and development. Then, it incurs high maintenance and labor cost. To solve the actual engineering problems, this paper presents a decentralized, flat-structured control method for HVAC system. It is based on intelligent nodes (CPN) to realize device self-identification and automatic networking. Moreover, using the developed decentralized control algorithm APP, the intelligent nodes can negotiate with each other to achieve global optimization control. It reduces the workload of wiring, configuring and commissioning, and it can be easily applied to other HVAC systems. The application of an actual decentralized HVAC automation system proved that the proved method is energy efficiency, operability and cost savings.

The optimized decentralized control for equipment of chiller group, water pump group, and cooling tower group is gained. However, the mechanisms among different device groups, like how the chiller group and water chill group work together to achieve a high energy efficiency, should be studied in the future. In addition, in the future research, long term data will be collected to further verify the effectiveness and energy saving of the control system.

Author Contributions: All the authors contributed to this study. D.H.: conceptualization, methodology, writing of the original draft; Q.X.: funding acquisition, project administration, supervision; X.Z.: writing of review and editing; Y.D. and Z.J.: investigation, software and validation.

Funding: This research was supported by Chongqing Technological Innovation and Application Demonstration Project under grant number cstc2018jszx-cyzdX0057 and National Natural Science Foundation of China under grant number 61473050 .

Conflicts of Interest: The authors declare no conflict of interest.

\section{Nomenclature}

$\begin{array}{llll}T & \text { Temperature } & \text { CPN } & \text { Computing process node } \\ \text { COP } & \text { Coefficient of performance } & \text { APP } & \text { Application } \\ \text { PLR } & \text { Partial load ratio } & a, b, c, d, j, k, l, s \text { Constant coefficient } \\ n & \text { Pump running speed } & & \\ H & \text { Pump head } & \text { Subscript } & \\ Q & \text { Pump flow rate } & \text { lea } & \text { Leaving the evaporator } \\ w & \text { Pump speed ratio } & \text { ent } & \text { Entering the condenser } \\ \eta & \text { Mechanical efficiency } & \text { act } & \text { Actual } \\ m & \text { Mass flow rate } & \text { rat } & \text { Rated } \\ \varepsilon & \text { Expected efficiency } & \text { wat } & \text { Water } \\ \Delta & \text { Demand deviation } & \text { out } & \text { Output } \\ \delta & \text { Set calculation precision } & \text { min } & \text { Minimum } \\ G & \text { Water flow rate } & \text { max } & \text { Maximum }\end{array}$

\section{References}

1. Lam, J.C. Energy analysis of commercial buildings in subtropical climates. Build. Environ. 2000, 35, 19-26. [CrossRef]

2. Lin, C.; Liu, H.; Tseng, K.; Lin, S. Heating, Ventilation, and Air Conditioning System Optimization Control Strategy Involving Fan Coil Unit Temperature Control. Appl. Sci. 2019, 9, 2391. [CrossRef]

3. Lu, S.; Wang, W.; Wang, S.; Cochran Hameen, E. Thermal Comfort-Based Personalized Models with Non-Intrusive Sensing Technique in Office Buildings. Appl. Sci. 2019, 9, 1768. [CrossRef]

4. Ma, Z.; Wang, S. Supervisory and optimal control of central chiller plants using simplified adaptive models and genetic algorithm. Appl. Energy 2011, 88, 198-211. [CrossRef]

5. Bortoni, E.D.C.; Almeida, R.A.D.; Viana, A.N.C. Optimization of parallel variable-speed-driven centrifugal pumps operation. Energy Effic. 2008, 1, 167-173. [CrossRef]

6. Wang, J.Y.; Chang, T.P.; Chen, J.S. An enhanced genetic algorithm for biobjective pump scheduling in water supply. Expert Syst. Appl. 2009, 36, 10249-10258. [CrossRef] 
7. Tianyi, Z.; Jili, Z.; Liangdong, M. On-line optimization control method based on extreme value analysis for parallel variable-frequency hydraulic pumps in central air-conditioning systems. Build. Environ. 2012, 47, 330-338. [CrossRef]

8. Westerlund, T.; Pettersson, F.; Grossmann, I.E. Optimization of pump configurations as a MINLP problem. Comput. Chem. Eng. 1994, 18, 845-858. [CrossRef]

9. Chang, Y.C. Optimal chiller loading by evolution strategy for saving energy. Energy Build. 2007, 39, 437-444. [CrossRef]

10. Torzhkov, A.; Sharma, P.; Li, C.; Toso, R.; Chakraborty, A. Chiller Plant Optimization-An Integrated Optimization Approach for Chiller Sequencing and Control. In Proceedings of the 2010 IEEE Conference on Decision and Control (CDC), Atlanta, GA, USA, 15-17 December 2010; pp. 2741-2746.

11. Chang, Y.C.; Lin, F.A.; Lin, C.H. Optimal chiller sequencing by branch and bound method for saving energy. Energy Convers. Manag. 2005, 46, 2158-2172. [CrossRef]

12. Lee, W.S.; Lin, L.C. Optimal chiller loading by particle swarm algorithm for reducing energy consumption. Appl. Therm. Eng. 2009, 29, 1730-1734. [CrossRef]

13. Chang, Y.C. An innovative approach for demand side management-optimal chiller loading by simulated annealing. Energy 2006, 31, 1883-1896. [CrossRef]

14. Zhang, Y.; Zhang, H.; Wang, Y.; You, S.; Zheng, W. Optimal configuration and operating condition of counter flow cooling towers using particle swarm optimization algorithm. Appl. Therm. Eng. 2019, 151, 318-327. [CrossRef]

15. Singh, K.; Das, R. An improved constrained inverse optimization method for mechanical draft cooling towers. Appl. Therm. Eng. 2017, 114, 573-582. [CrossRef]

16. Serna-Gonzalez, M.; Ponce-Ortega, J.M.; Jiménez-Gutiérrez, A. MINLP optimization of mechanical draft counter flow wet-cooling towers. Chem. Eng. Res. Des. 2010, 88, 614-625. [CrossRef]

17. Rao, R.V.; Patel, V.K. Optimization of mechanical draft counter flow wet-cooling tower using artificial bee colony algorithm. Energy Convers. Manag. 2011, 52, 2611-2622. [CrossRef]

18. Yao, F.; Sun, H. Optimal Control in Variable-Speed Pumping Stations. In Proceedings of the 2011 IEEE International Conference on Mechatronics and Automation, Beijing, China, 7-10 August 2011; pp. 2397-2401.

19. Dai, Y.; Jiang, Z.; Chen, P.; Jiang, Y. Speed optimization control of parallel-connected variable speed pumps. ASHRAE Trans. 2015, 121, 395-402.

20. Dai, Y.; Jiang, Z.; Xin, S.; Chen, P.; Li, S. Optimal Control of Variable Speed Parallel-Connected Pumps. In Proceedings of the 13th International Conference on Indoor Air Quality and Climate, Hong Kong, China, 1 January 2014; pp. 87-94.

21. Kallesøe, C.; Aarestrup, J.; Rokkjær, K. Energy optimization for booster sets. World Pumps. 2011, 12, 24-30. [CrossRef]

22. Karaca, M.; Aydin, M. Efficient driving at variable speeds. World Pumps. 2013, 4, 38-41. [CrossRef]

23. Gordon, J.M.; Ng, K.C. Cool Thermodynamics; Cambridge Int. Science Publishing: Great Abington, Cambridge, UK, 2000.

24. Jiang, Z.; Dai, Y. A decentralized, flat-structured building automation system. Energy Procedia 2017, 122, 68-73. [CrossRef]

25. Cai, H.; Wei, Q. Operation Performance Evaluation and Energy Saving Diagnosis of Water Chillers. In Proceedings of the 2008 China Annual Academic Conference on HVAC Refrigeration, Chongqing, China, 4 November 2008; pp. 106-111.

26. Liu, Z. Modeling and Energy Saving Optimization of Air Conditioning System in Public Buildings. Ph.D. Thesis, Tsinghua University, Beijing, China, May 2015.

27. Liu, Z.; Chen, X.; Jiang, Z.; Qiao, L.; Guan, X. Asynchronous Latency Analysis on Decentralized Iterative Algorithms for Large Scale Networked Systems. In Proceedings of the 32nd Chinese Control Conference, Xi'an, China, 26-28 July 2013; pp. 6900-6905.

28. Dai, Y.; Jiang, Z.; Shen, Q.; Chen, P.; Wang, S.; Jiang, Y. A decentralized algorithm for optimal distribution in HVAC systems. Build. Environ. 2016, 95, 21-31. [CrossRef]

(C) 2019 by the authors. Licensee MDPI, Basel, Switzerland. This article is an open access article distributed under the terms and conditions of the Creative Commons Attribution (CC BY) license (http://creativecommons.org/licenses/by/4.0/). 